\title{
Transiting Disintegrating Planetary Debris around WD 1145+017
}

\author{
Andrew Vanderburg and Saul A. Rappaport
}

\begin{abstract}
More than a decade after astronomers realized that disrupted planetary material likely pollutes the surfaces of many white dwarf stars, the discovery of transiting debris orbiting the white dwarf WD 1145+017 has opened the door to new explorations of this process. We describe the observational evidence for transiting planetary material and the current theoretical understanding (and in some cases lack thereof) of the phenomenon.
\end{abstract}

\section{Overview}

A bit more than a decade after astronomers first began to suspect that white dwarf stars occasionally disrupt and accrete asteroids and small planets from their primordial planetary systems (Debes and Sigurdsson 2002; Jura 2003), an impressive body of evidence had emerged in support of this scenario. In early 2015, it was known that (a) a large fraction of white dwarf stars (between $25 \%$ and 50\%) are "polluted" with trace amounts of elements like silicon, iron, calcium and magnesium in their atmospheres (Zuckerman et al. 2010, Koester et al. 2014), (b) many of these polluted white dwarfs also showed evidence of warm rocky material orbiting the star in a debris disk (Barber et al. 2012), and (c) the abundance ratios of heavy elements in the atmospheres of white dwarfs very closely matched the abundance patterns in rocky bodies in the solar system (Zuckerman et al. 2007; Farihi et al. 2013).

The generally accepted explanation for these observations was that these polluted white dwarfs host planetary systems which at least partially survived the white dwarf progenitor's evolution off the main sequence. The host star's evolution was not without ill effects: as the host shed its outer layers and began to cool and contract into a white dwarf, the star's mass loss caused changes to the planetary system's dynamics. Numerical simulations have shown that planetary systems whose host stars have undergone this type of mass loss can occasionally perturb small planets or asteroids into highly eccentric orbits which can occasionally have periastron passages close enough to the host star (by this time a white dwarf) to be tidally disrupted. The planetary, lunar (Payne et al.|2016), or asteroidal remnants would then be pulverized into a fine dust (causing the infrared excesses observed around many polluted white dwarf stars) and slowly accreted onto the white dwarf's surface, where the constituent elements would manifest themselves by the presence of spectral lines.

However, the evidence for this scenario was entirely circumstantial, relying on analysis of the after-effects of planetary disruption. There were occasional detections of transient events indicating possible tidal disruptions in progress (Del Santo et al. 2014, Xu and Jura 2014), but no unambiguous detections of disintegrating rocky material until the discovery of transits around a polluted white dwarf called WD 1145+017. For the first time, astronomers had definitively observed the transient process of a white dwarf tidally disrupting a large rocky body (planet, moon, or asteroid),

Andrew Vanderburg

Harvard-Smithsonian Center for Astrophysics, Cambridge, MA 02138

Current address: Department of Astronomy, The University of Texas at Austin, Austin, TX 78712, USA

e-mail: avanderburglutexas.edu

Saul A. Rappaport

Massachusetts Institute of Technology, Cambridge, MA 02139, e-mail: sar@mit.edu 
in real time. WD 1145+017 has provided the strongest evidence yet that white dwarfs disrupt their planetary systems, and has given astronomers new ways to study and constrain models of the process.

\section{Discovery Observations}

WD 1145-017 was first identified as a somewhat anonymous white dwarf in 1991 by Berg et al. (1992), who used lowresolution spectroscopy to classify it as a helium-envelope white dwarf. This classification was confirmed by Friedrich et al. (2000), although neither groups' classification spectra were strong enough nor taken at high enough spectral resolution to detect any features besides the deep and broad helium lines. Little attention was given to WD $1145+017$ until 2014, when it fell in one of the fields of view of NASA's Kepler Observatory in its extended K2 mission. During the interval between June and August 2014, K2 observed the field containing WD $1145+017$ (along with 20,000 other stars) for a bit less than 80 days.

Once the K2 data were publicly released, Vanderburg et al. (2015) searched through the data looking for planetary transits. The majority of the stars observed by K2 were main-sequence stars, typically the mass of the sun or lower. However, a handful of other objects, including about 150 white dwarf stars, were proposed by various groups and were observed by $\mathrm{K} 2$ as well.

A periodogram search of the K2 data revealed about 80 'ordinary' transiting planet candidates around main sequence stars, as well as a strong transit-like signal at a period of 4.5 hours from WD $1145+017$. The transit profile was broad (occupying nearly $30 \%$ of an orbital cycle), had a profile that was unlike that of a typical hard-body transit, and was variable in depth. The periodogram and fold about the 4.4989-hour period are shown in Fig. 1 . A closer analysis of the K2 light curve also revealed evidence for five other significant periodicities with periods between 4.55 and 4.86 hours (designated Periods B through F), suggesting multiple bodies in close orbits. These other periodicities can be seen in the Lomb-Scargle periodogram in Fig. 1, and the corresponding folded transit profiles are shown in the panels below the periodogram.

A significant limitation of the K2 data was its time sampling. For bandwidth reasons, Kepler only can store photometric data for the majority of the targets it observes, including WD 1145+017, after an integration time of 30 minutes. Because the transit signal around WD $1145+017$ had a period of 4.5 hours, K2 only recorded nine photometric measurements per orbit. This limitation is particularly unfortunate for studying objects transiting or eclipsing white dwarfs, because the host star's small size means transits and eclipses happen on timescales of about 1 minute, rather than the hour to day timescales for transits of main sequence stars. Such rapid transit features are totally smeared out by the 30-minute $\mathrm{K} 2$ integrations.

Motivated by the very short orbital periods found in the K2 data, Vanderburg et al. (2015) began photometric observations of WD 1145+017 using small ground-based telescopes at rapid cadence to confirm and better resolve the transits. After a few nights of high-cadence photometric observations, several things became clear. WD $1145+017$ was indeed being transited by objects in a $\simeq 4.5$ hour orbital period, but the transits were not always present. The transits were deep, up to $40 \%$ of the star's flux, and lasted about 5 minutes - too deep and short in duration to be a transit of anything other than a white dwarf, but too long to be the transit of a small solid body across the white dwarf star (see Fig. 2). The transits were asymmetric with a fast ingress time and slow egress time - similar to transits of disintegrating planets observed around main sequence stars by Kepler (see see van Lieshout and Rappaport, "Disintegrating Rocky Exoplanets"; this Handbook). Finally, the transits did not always occur at the same phase of a 4.5 hour orbit. On two different nights, Vanderburg et al. (2015) observed two convincing transits separated by 4.5 hours, but the pairs of transits observed on each of these nights happened almost 180 degrees out of phase, with respect to a 4.5 hour orbit, further suggesting the possibility of multiple objects in orbit.

Medium resolution spectroscopy from the MMT Observatory yielded two additional important clues. First, there was no evidence for radial velocity variations over the 4.5 hour orbital periods (with a limit of $\sim 500 \mathrm{~m} \mathrm{~s}^{-1}$ ), confirming that any bodies transiting WD $1145+017$ had to be of planetary mass or less. More importantly, the MMT spectrum revealed that WD 1145+017 exhibited absorption lines from elements heavier than hydrogen and helium, including magnesium, aluminum, silicon, calcium, iron, and nickel. WD 1145+017 is therefore a "polluted" white dwarf - a member of the class of white dwarfs believed to have accreted disrupted planetary material. Data from NASA's WISE spacecraft show evidence for infrared excess emission, another hallmark of polluted white dwarfs and evidence for disrupted planetary material in orbit of the white dwarf. 
Vanderburg et al. (2015) interpreted the transits of WD $1145+017$ as being produced by dust clouds. This inference was made by analogy with a similar phenomenon of so-called 'disintegrating' planets that are found transiting mainsequence stars and which appear to exhibit dusty tails ("Disintegrating Rocky Exoplanets"; this Handbook). These objects, including KIC 12557548 (Rappaport et al. 2012), KOI 2700 (Rappaport et al. 2014), and K2-22 (SanchisOjeda et al. 2015), show similar features to the objects transiting WD 1145+017, including asymmetric transits with rapidly varying transit depths. The disintegrating planets are all in short-period orbits around their hosts stars, and are highly irradiated, to the point where rocky minerals would likely sublimate rapidly. Perez-Becker and Chiang (2013) showed that a small, sub-Mercury sized object orbiting close to a host main-sequence star could undergo rapid mass loss as rocky material sublimates due to the high irradiation environment, and flows away from the planet in a Parker-type thermal wind.

While the idea of small disintegrating rocky objects with dusty effluents causing the transits is at least partially and qualitatively successful, Vanderburg et al. (2015) freely admitted that the observations of WD 1145+017 do not support the exact scenario seen in the systems orbiting main-sequence stars. In particular, there have been no detections of phase shifts in the transits of disintegrating planets around main-sequence stars - when the transits of these canonical disintegrating planets appear, they always happen at the same orbital phase. The cause of the phase shifts between the different sets of transits seen by Vanderburg et al. (2015) was clarified by Croll et al. (2015) who, acting on news of the detection of transits of WD 1145+017, obtained a considerably larger set of ground-based follow-up data than Vanderburg et al. (2015) in May 2015. Over the course of 32 hours of observations, Croll et al. (2015) detected nine transits. A blind periodicity search for these transits revealed that many of the transits seemed to recur with an orbital period about 30 seconds shorter than the dominant period detected by Vanderburg et al. (2015) in K2 data, but that the recurring transits appeared to be scattered over a range of orbital phases. Croll et al. (2015) interpreted these transits as being caused by many different discrete bodies in almost identical 4.5 hour orbits, explaining the phase shifts seen between the sets of transits detected by Vanderburg et al. (2015).

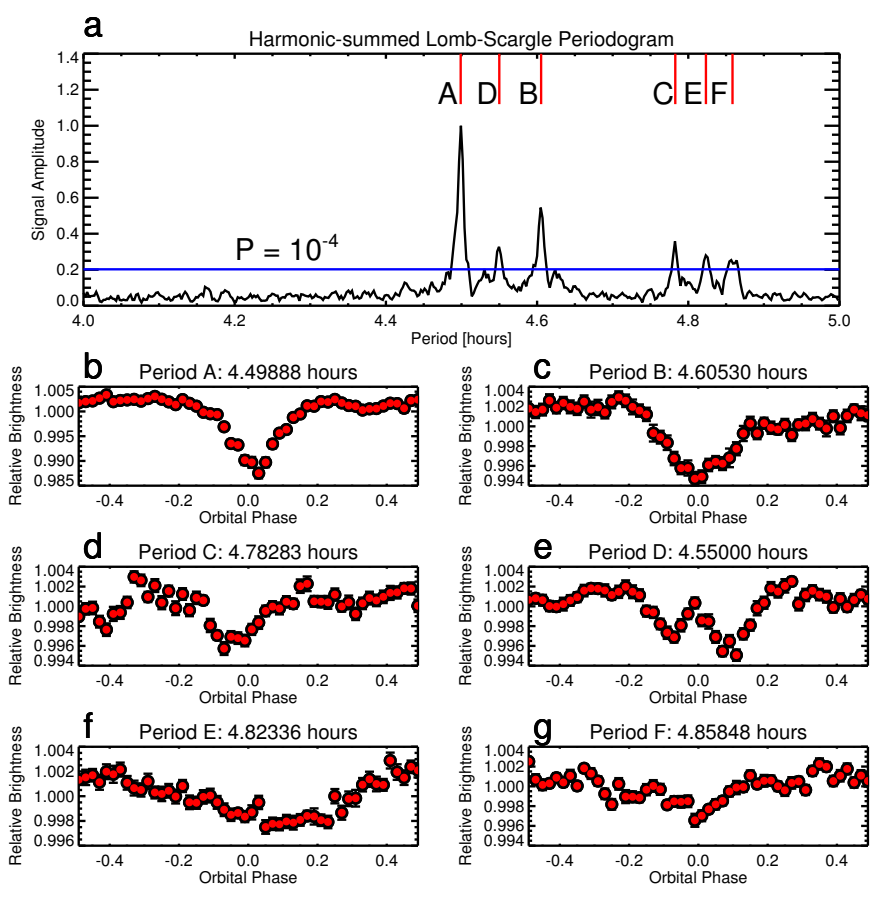

Fig. 1 K2 discovery observations of WD $1145+017$ (Vanderburg et al. 2015). Top panel: Lomb-Scargle periodogram with amplitudes of the first 3 harmonics summed. Six significant and distinct peaks are identified. Bottom panel: Folded lightcurve for each of the detected periodicities. The corresponding fold period is written above each panel.

Meanwhile, an independent group led by Siyi Xu also found their attention drawn to WD 1145+017. Xu et al.(2016) were conducting a spectroscopic survey of white dwarfs with excess infrared emission at high spectral resolution with the goal of detecting heavy elements in white dwarf spectra to learn about the compositions of small extrasolar 


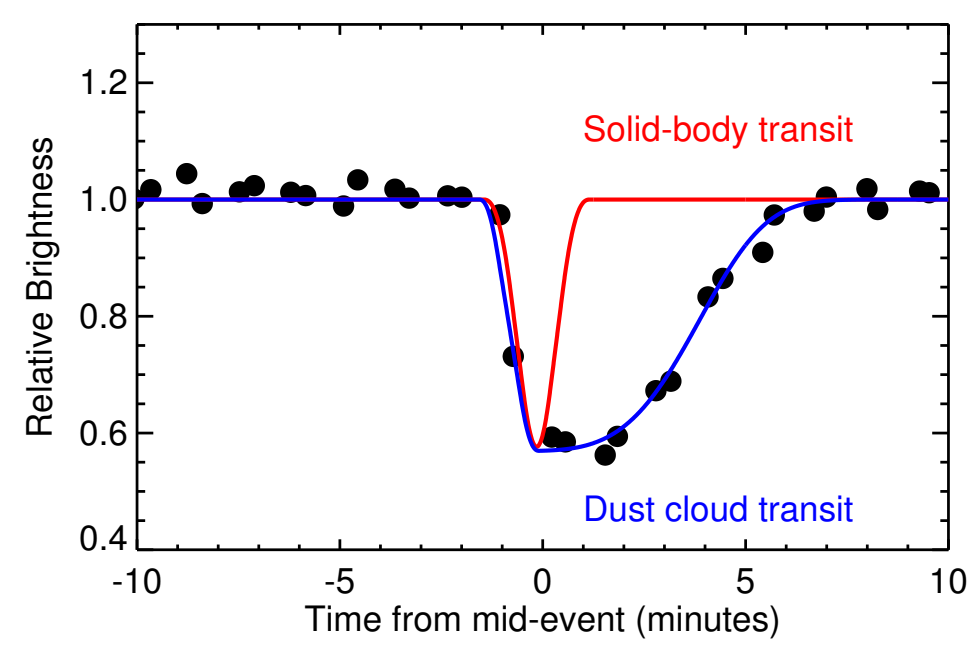

Fig. 2 Ground-based observations of two transits by Vanderburg et al. (2015), compared to a model transit of a solid body (red) and a dust cloud (blue). We note that the transit shapes do not always have the short ingress-long egress asymmetry. Follow-up observations have shown that while there is some preference for this shape, symmetric transits and long ingress-short egress asymmetries are common as well.

asteroids or planets. On 15 April 2015, only a few hours after Vanderburg et al. (2015) detected the first set of transits in their ground-based follow-up, Xu et al. (2016) obtained a high signal-to-noise spectrum of WD $1145+017$ with the High Resolution Echelle Spectrometer (HIRES) at Keck Observatory. As Xu et al. (2016) expected from their detection of near infrared excess emission, the spectrum revealed the presence of numerous absorption features corresponding to elements like iron, silicon, nickel, magnesium, and calcium. Xu et al. (2016) were surprised, however, to find broad absorption features near many of the detected metal absorption lines, presumably caused by circumstellar gas orbiting WD 1145+017. Illustrative composite spectra (i.e., summed over 5 different metal lines) are shown in Fig. 3 for the 2015 April observations as well as from a follow-up observation from 2016 February. The circumstellar features are broad (with line widths up to $300 \mathrm{~km} \mathrm{~s}^{-1}$ ) and deep (obscuring up to $30 \%$ of the star's flux at those wavelengths), and the features are visible in lines from ionized states of iron, magnesium, chromium, titanium, calcium, manganese, and nickel.

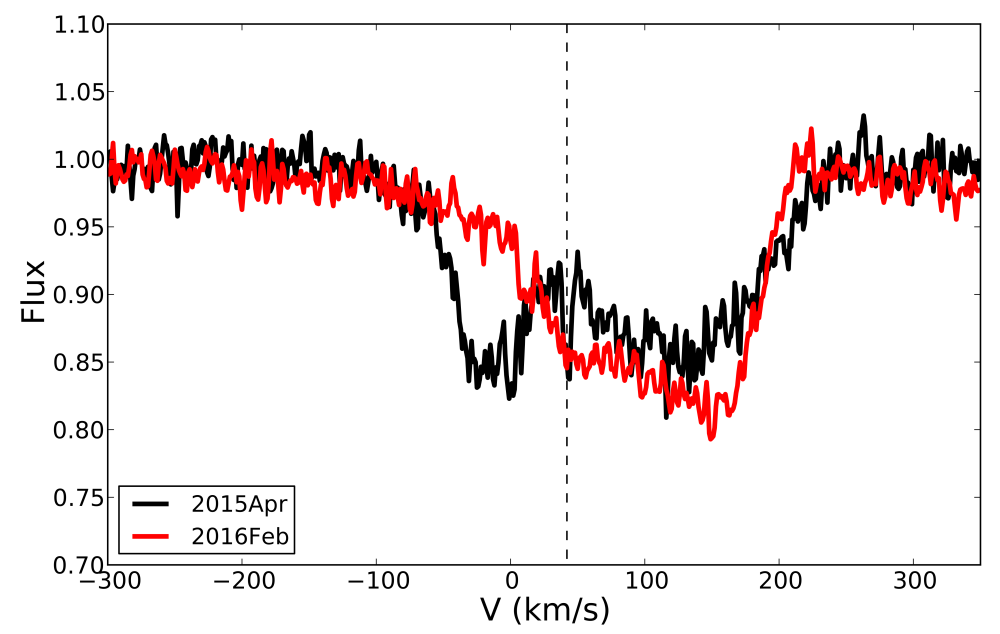

Fig. 3 Illustrative composite spectra (i.e., summed over 5 different metal lines) for the 2015 April observations as well as from a follow-up observation from 2016 February (Siyi Xu, 2016, private communication.). Note the broad line widths of $\sim 300 \mathrm{~km} \mathrm{~s}^{-1}$. The offset of the dashed vertical line from zero indicates the gravitational redshift from the surface of the white dwarf. 
The circumstellar absorption features found by Xu et al. (2016) are unique among all other polluted white dwarfs. Some white dwarfs show circumstellar gas in emission (Gänsicke et al. 2006, Manser et al. 2016) indicating hot gas disks (not necessarily viewed edge-on), and other white dwarfs show weak circumstellar absorption features in ultraviolet spectra and optical spectra (Debes et al.|2012a, Gänsicke et al.|2012), but none shows circumstellar absorption at the strengths seen around WD 1145+017. The discovery of circumstellar gas absorption at WD $1145+017$ is independent confirmation of the presence of material orbiting close to the white dwarf between the star and our vantage point on Earth. The same favorable orbital inclination of the WD 1145+017 disrupted planetary system, i.e., near $90^{\circ}$, is required to see both the transits and the circumstellar gas absorption.

\section{Ground-based follow-up observations}

\section{Ground-based photometric monitoring}

The first extensive and systematic ground-based photometric observations of WD 1145+017 commenced in 2015 November (Gänsicke et al. 2016; Rappaport et al. 2016). Gänsicke et al. (2016) photometrically observed WD $1145+017$ during 15 nights in 2015 November using $2.4 \mathrm{~m}$ and $1 \mathrm{~m}$ telescopes. The results are shown in Fig. 4. Several distinct dips can be seen over the course of a single orbit, with some as deep as 50\%. Averaged over the orbit, as much as $\sim 11 \%$ of the flux is removed by the dips. It is quite apparent from this result that the source was much more 'active', in the sense of having more and deeper dips, during this period than during the $\mathrm{K} 2$ and the initial ground-based followup observations (Vanderburg et al. 2015, Croll et al. 2015).

A number of the dips seen in Fig. 4 could be tracked from night to night, thereby allowing for more precise periods to be derived. The periods found by Gänsicke et al. (2016) range from 4.491 to 4.495 hours. These differ by between $0.1 \%$ and $0.2 \%$ from the K2 ' $\mathrm{A}$ ' period of 4.4989 hours. No sign of the K2 'B' through ' $\mathrm{F}$ ' periods was found in the Gänsicke et al. (2016) data. However, if the dips at these latter periods had remained at the same depths found in the K2 observations (fractions of a percent) they could not have been detected. Thus, it appears that WD 1145+017 was mostly active near the 'A' period in 2015 November, but with much greater dip depths.

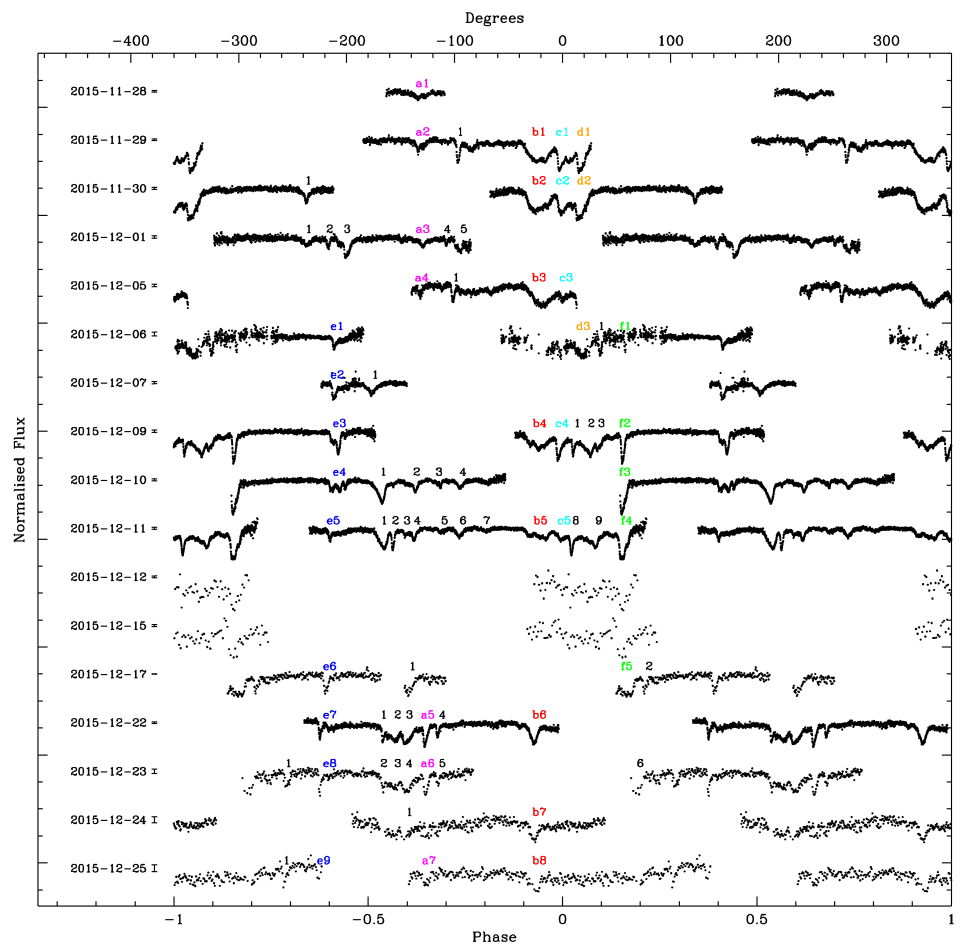

Fig. 4 Photometric monitoring of WD $1145+017$ for an interval of a month using a 2.4-m telescope (from Gänsicke et al. 2016). The phasing of the diagram uses a period of $4.4930 \mathrm{hr}$, approximately the same as the 'A fragments' detected by ground-based observations. 


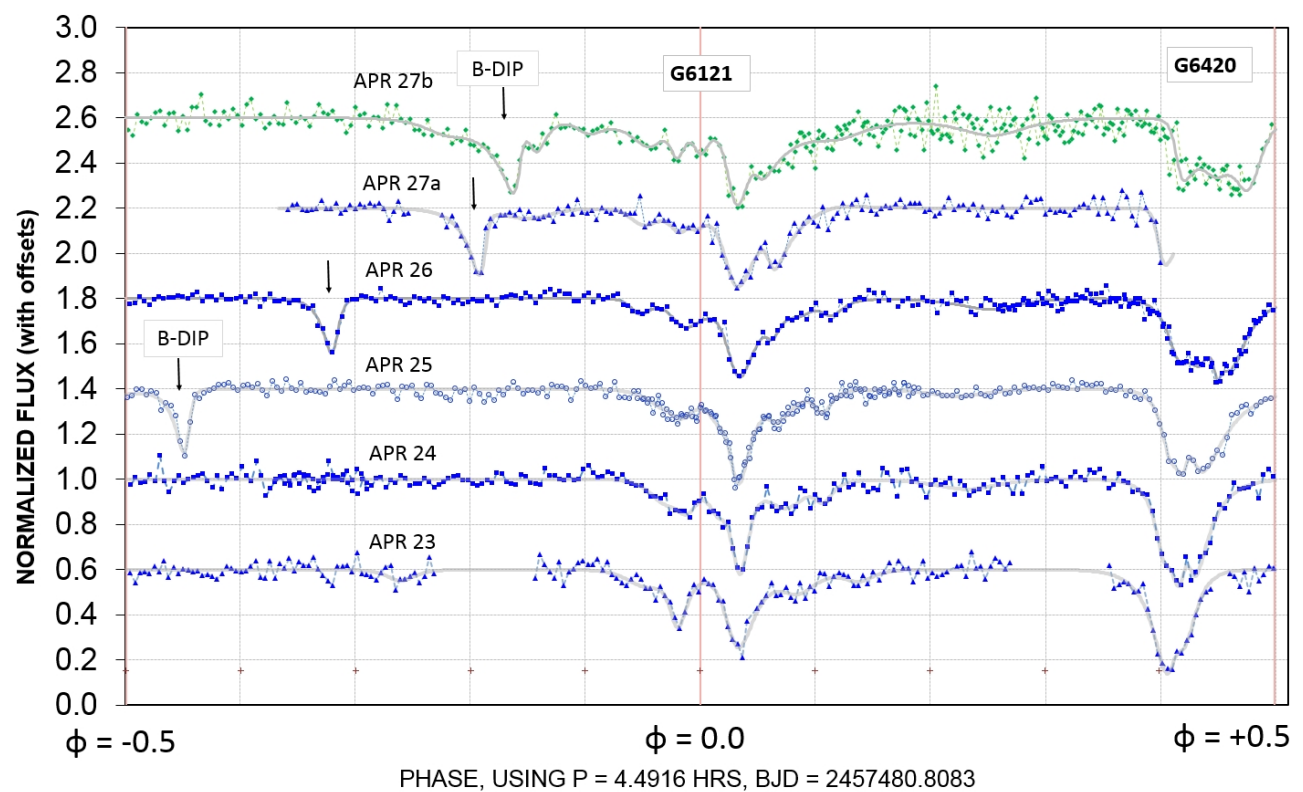

Fig. 5 Stack of 6 lightcurves for WD 1145+017 reported by Gary et al. (2016) from 2016 April. The first 5 lightcurves (blue) are from the IAC80 $32^{\prime \prime}$ telescope and the last one (green) is from a $20^{\prime \prime}$ telescope.

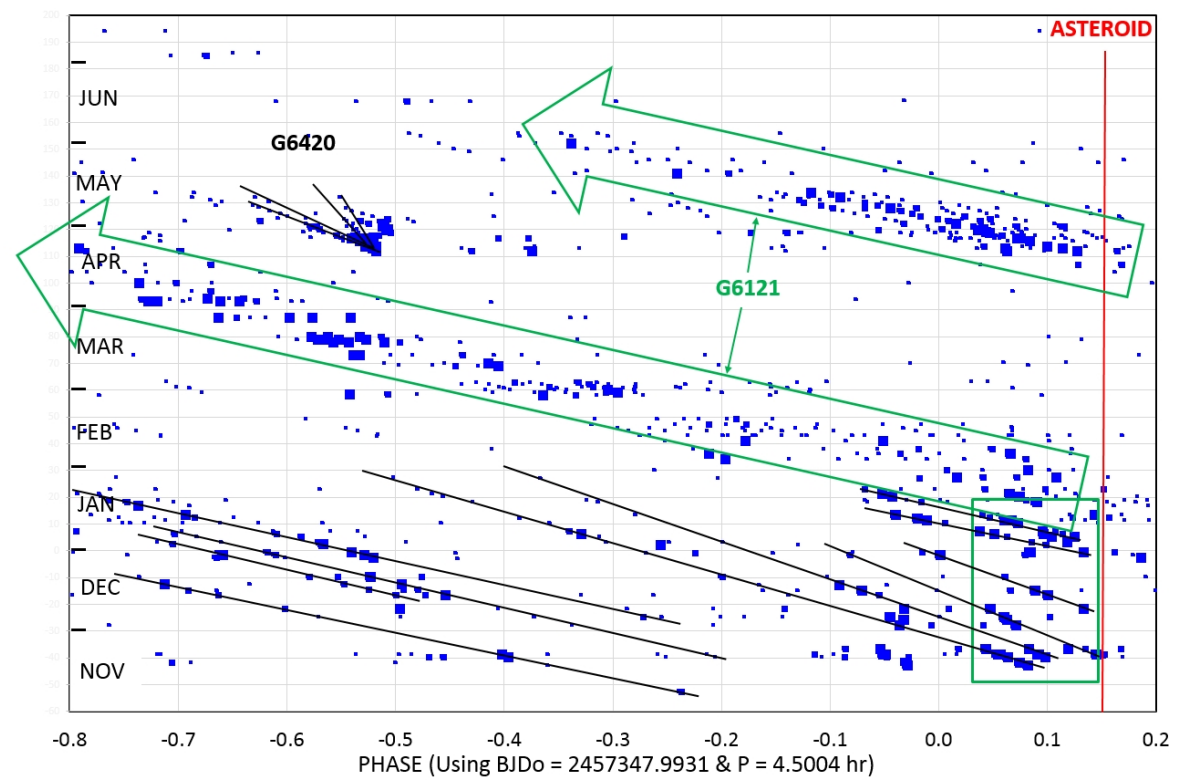

Fig. 6 'Waterfall' diagram for WD 1145+017 of the dips recorded over 8 months from 2015 November through 2016 June (Gary et al. 2016). The phasing here is based on the 'A-asteroid' period of $4.5004 \mathrm{hr}$, essentially the same as found with $\mathrm{K} 2$.

Starting simultaneously with the Gänsicke et al. (2016) observations, Rappaport et al. (2016) and Gary et al. (2016) began a much longer monitoring campaign of WD $1145+017$ using small optical telescopes in the $30-80 \mathrm{~cm}$ range. A typical set of lightcurves from five sequential nights is shown in Fig. 5. Here the data are stacked vertically and phased to an assumed period of 4.4916 hours. The dips near phases 0 and 0.4 are nearly repeatable over the five nights. However, there is clearly an additional dip that is moving quickly in phase, labeled 'B-dip'. This dip feature was associated by Gary et al. (2016) with an object orbiting at the K2 'B' period. 
Gary et al. (2016) (see also Rappaport et al. 2016) found it useful to construct so-called 'waterfall' diagrams to display the complicated temporal behavior of the dip evolution. These diagrams are produced as follows. The dips are all formally fit with a simple analytic function (in this case an asymmetric hyperbolic secant, 'AHS'; see Rappaport et al.2016), whose fitted parameters are the depth, center time, and ingress and egress times. One can then use these parameters to plot the kind of 'waterfall' diagram shown in Fig. 6. In that diagram, each dip is represented by a rectangular bar, with orbital phase during a single night plotted in the horizontal direction, and observation night plotted vertically. The depth of the dip is proportional to the thickness of the bar, and the duration is equal to the length of the bar. This particular plot is phased to an assumed period of $4.500 \mathrm{hr}$, the dominant mean period found by Rappaport et al. (2016).

As one can see from Fig. 6 there is a collection of dips between phases 0.03 and 0.15 during 2015 November and December. After that, and for the ensuing $\sim 5$ months, the orbiting objects started drifting in phase (the group labeled 'G6121'). The periods associated with these drifting features in the lightcurve were in the range 4.491 to 4.495 hours, in basic accord with those found by Gänsicke et al. (2016).

We summarize here some of what was learned after 8 months of monitoring WD 1145+017 during the 2015-2016 observing season. First, the activity level of transits was quite high with dips obscuring as much as $10 \%$ of the flux averaged around the orbit. This is at least an order of magnitude larger than during the $\mathrm{K} 2$ discovery period. The source dust activity level is shown as a function of time in Fig. 7. Second, numerous different periods are seen between 4.490 to 4.500 hour, possibly indicating a dozen different bodies orbiting and emitting dusty effluents. These are all within $0.2 \%$ of the K2 'A' period. Only one of the other five K2 periods, i.e., the 'B' period, became active enough to detect with the small monitoring telescopes, but remained detectable for only 2-3 weeks. Third, in all photometric monitoring to date, the transits have never been deeper than $60 \%$ of the star's total flux. Finally, we can see from Fig. 7 that the source activity at the start of the 2016-2017 observing season is now even higher than during all of the previous season, and has recently obscured as much as $17 \%$ of the orbit-averaged flux, but is currently declining to lower values (B. Gary, private communication).

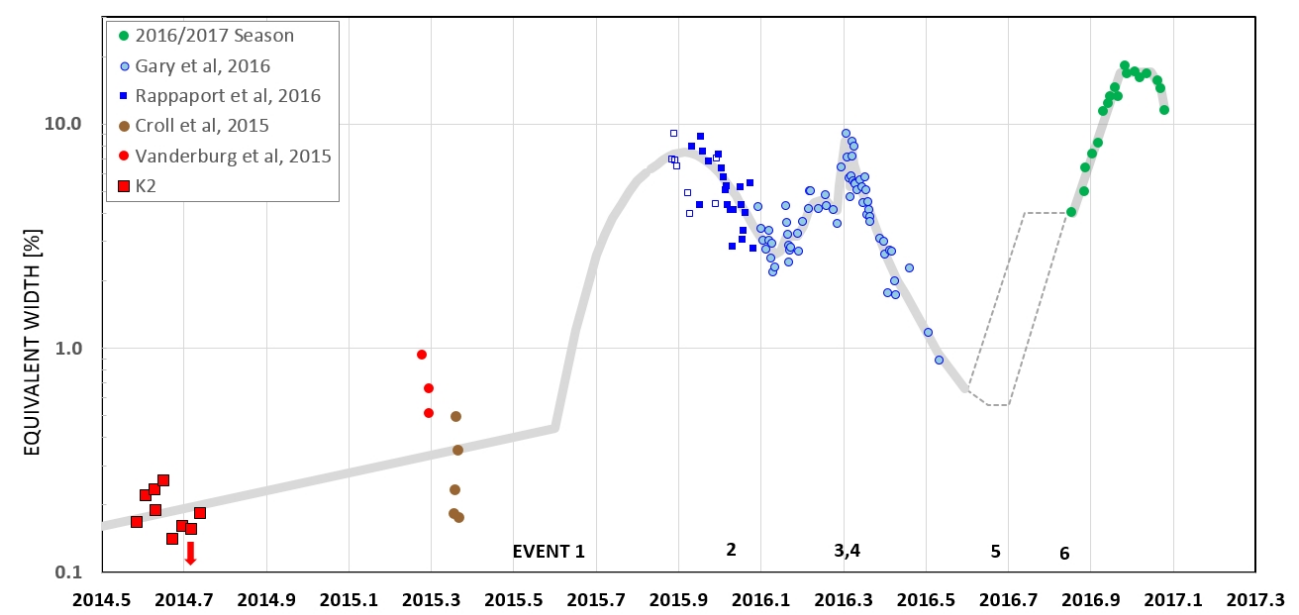

Fig. 7 Activity level of WD $1145+017$ since its discovery with K2 (from Gary et al. 2016 and private communication). The 'activity' is defined as the dip depths averaged over an orbital cycle.

Finally in regard to the 'A' period, the ground-based observations from Croll et al. (2015), Gänsicke et al. (2016), Rappaport et al. (2016), and Gary et al. (2016) all find a period that is slightly, but significantly, shorter than the 4.5 hour A-period found with K2. Rappaport et al. (2016) proposed a model where a large asteroid, about 1/10 the mass of Ceres occasionally released fragments into slightly shorter period orbits. Gurri et al. (2017) simulated the subsequent interactions of multiple fragments in such a shorter-period orbit with the parent asteroid as well as amongst themselves, while Veras et al. (2017) showed explicitly how a rubble pile near the Roche limit discharges fragments into shorter (as well as longer) period orbits. However, since early 2016, there has been no additional observational evidence that the 4.5 hour K2 A-period is persistent or "special," raising some questions about this interpretation. 
Assuming that the dips in flux from WD 1145 are a result of dusty effluents from rocky bodies, it is important to learn what we can about the nature of the dust. Once the dust sublimates, its chemical composition, and by implication that of the planetesimal, can be investigated from its transmission spectrum as it crosses the face of the white dwarf (Xu et al.2016; see the Discovery Observation section). While the material is still in the form of dust grains, we are limited for now, to attempting to measure (i) its size distribution and (ii) some rough information about its chemical composition from both the grain scattering properties and lifetimes against sublimation.

Thus far, there have been several attempts to measure the colour-dependence of the dips in the visible (Croll et al. 2015; Alonso et al.|2016; Z. Berta-Thompson 2016, private communication) and in the NIR (Zhou et al.|2016). None of these has succeeded in finding a difference in transit depth with wavelength, and we can thereby set constraints on the dust grain sizes of $\sim 0.5 \mu \mathrm{m}$ in the visible band and $\sim 0.8 \mu \mathrm{m}$ in the NIR. A good example of this type of measurement is shown in Fig. 8 . There are four superposed traces of the flux from WD1145 in different wavebands as a function of time over more than half an orbital cycle showing several dip features. The four traces are in bands covering $480 \mathrm{~nm}$ to $920 \mathrm{~nm}$, and there are no systematic differences observed among the different bands. Alonso et al. (2016) showed that the depths of these transits were the same to within a statistical precision of $\sim 1 \%$ from which they were able to rule out small particles below $\sim 0.5 \mu \mathrm{m}$. This limit is derived from the fact that for particles whose sizes are greater than the observing wavelength, the scattering cross section quickly becomes equal to the geometric area of the dust grain. When that occurs, the scattering becomes independent of wavelength.

\section{Time-resolved spectroscopy}

Continued observations of the circumstellar gas absorption lines (Redfield et al. 2016, S. Xu 2016, private communication) show that the shape and intensity of these broad lines are highly variable on different timescales. One possible explanation for the broad and shifted absorption lines is a mildly eccentric ring of gas orbiting close to the white dwarf (see also Redfield et al. 2016). It can be shown that for a mildly eccentric orbit (i.e., with $e \lesssim 0.1$ ), the radial velocity of the gas as a function of its transverse location, $x$, across the face of the host star, is given by:

$$
v_{r} \simeq v_{0}(e \cos \omega-x / a) \simeq \frac{2030}{P_{\min }^{1 / 3}} e \cos \omega-\frac{900}{P_{\min }}\left(\frac{x}{R_{\mathrm{wd}}}\right) \mathrm{km} \mathrm{s}^{-1}
$$

where $\omega, v_{0}$, and $a$ are the argument of periastron, orbital speed, and semimajor axis for the orbiting ring of gas, respectively. The expression on the right is specifically for the WD $1145+017$ system, $P_{\min }$ is the orbital period expressed in units of minutes, and $R_{\mathrm{wd}}$ is the radius of the white dwarf. Thus, for example, if a line is observed with a range of wavelengths corresponding to radial velocities of -100 to $+200 \mathrm{~km} \mathrm{~s}^{-1}$, this yields $e \cos \omega \simeq 0.045$ and $P \simeq 6$ minutes.


Fig. 8 GTC lightcurves of WD $1145+017$ taken simultaneously in four wavebands and covering several dips (Alonso et al. 2016). The nearly identical dip profiles in the four bands can be used to constrain the dust grain sizes to $\gtrsim 0.5 \mu \mathrm{m}$. The divergence of the curves after phase 0.22 in the lower panel is due to atmospheric effects. 
Some Theoretical Considerations

The pollution of white dwarfs had been a long-standing problem in astrophysics, dating back to the 1970s (Fontaine and Michaud (1979), when it was realized that in order for heavy elements to appear in the spectra of white dwarfs, they must have been recently accreted from external sources (or they would have quickly sunk to the center of the white dwarfs, where they would be un-observable). The history of this problem is extensively discussed in "Characterizing Planetary Systems Around White Dwarfs" (B. Zuckerman and E. Young; this Handbook). In brief, the discovery of dusty debris disks near many polluted white dwarfs (Zuckerman and Becklin 1987; Becklin et al. 2005) and the fact that the abundances of heavy elements found in white dwarfs were quite similar to the elemental ratios in rocky solar system bodies (Zuckerman et al. 2007) led astronomers to believe that these heavy elements were likely the disrupted remains of rocky objects from the white dwarf progenitors' planetary systems.

\section{Origin of the Debris:}

Debes and Sigurdsson (2002) were among the first to suggest that heavy element pollution in white dwarfs was caused by the remnants of ancient planetary systems. Using analytic arguments and numerical studies, Debes and Sigurdsson (2002) showed that when an evolving star undergoes mass loss, previously stable planetary systems could become unstable to close encounters. The critical Hill separation for two equal-mass planets, $\Delta_{c}$, in terms of the semi major axes of two planets, $a_{1}$ and $a_{2}$, is given by:

$$
\Delta_{c}=\frac{a_{2}-a_{1}}{a_{1}} \simeq \sqrt{\frac{8}{3}\left(e_{1}^{2}+e_{2}^{2}\right)+9\left(\frac{m}{M}\right)^{2 / 3}}
$$

where $e_{1}$ and $e_{2}$ are the eccentricities of the two planets, $m$ is the mass of each of the two planets, and $M$ is the mass of the star. Debes and Sigurdsson (2002) noticed that when the stellar mass $M$ decreases (for a white dwarf progenitor, often by a factor of two or more), the critical separation increases, which can push previously stable systems into the unstable regime, rearranging the planetary system dynamically. This rearrangement, Debes and Sigurdsson (2002) speculated, could cause an increased rate of comets from the outer parts of the planetary system to be scattered inwards to close encounters with the white dwarf.

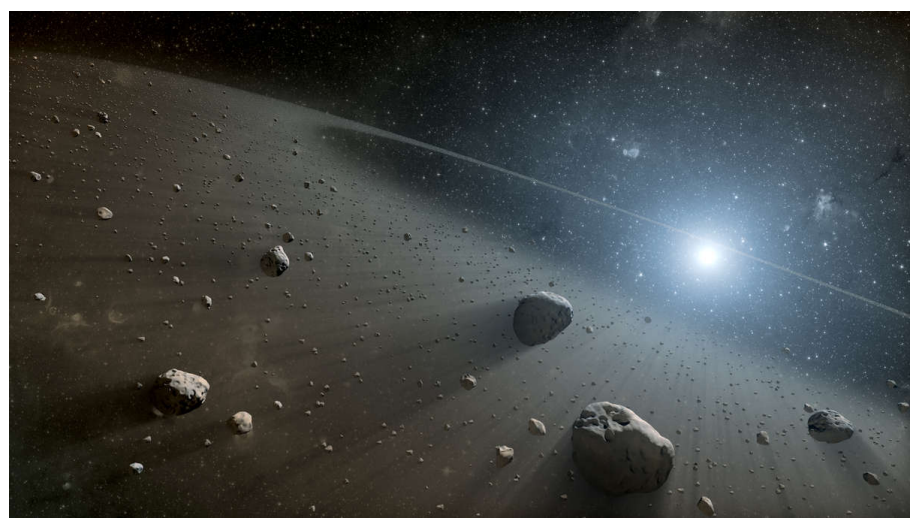

Fig. 9 Artist conception of what the WD 1145+017 debris disk system might look like as viewed from one of the orbiting asteroids. Image credit: NASA/JPL-Caltech

Since then, other mechanisms have been proposed to bring planetary material to close encounters with the host white dwarfs. Debes et al. (2012b) showed that stellar mass loss can sweep asteroids into mean motion resonances with giant planets (akin to Jupiter), where the asteroids are perturbed into highly eccentric orbits and close encounters with the white dwarf. Similarly, Bonsor et al. (2011) showed that stellar mass loss in a planetary system can perturb objects from an exo-Kuiper belt close to the white dwarf. Additionally, numerical studies have shown that for systems that border on Hill instability, increasing the Hill radii of the planets can in many cases cause the planetary systems themselves to become unstable, and undergo strong dynamical interactions on relatively short timescales, leading to close periastron passages with the white dwarf (Veras et al. 2013, 2016b). 
Roche Limit:

After rocky bodies have been dynamically perturbed too close to the host white dwarf, they are subject to tidal breakup. The famous result of Edouard Roche, cast in the context of bodies orbiting WD 1145+017 can be written as:

$$
s_{\text {crit }}=\xi R_{*}\left(\rho_{*} / \rho_{p}\right)^{1 / 3}
$$

where $s_{\text {crit }}$ is the critical distance from a star of density $\rho_{*}$ that a planetesimal of uniform density $\rho_{p}$ can come before it is tidally split. For Roche's original problem he found that the constant $\xi$ had a value of 2.45 . Newer treatments of the 'tidal splitting' problem have been carried out since then, and many of these are summarized by Davidsson(1999; see also Holsapple and Michel 2006). Taking into account such issues as the criteria for actually splitting the body into separated pieces; what trajectory the body is on; whether the body is corotating with its orbit; and the role that material strength plays, $\xi$ is found to lie closer to the range of $1.2 \lesssim \xi \lesssim 1.7$. There is also the issue of how centrally concentrated the mass of the planetesimal is, e.g., the case where its central density may be considerably higher than in its mantle. In the limiting (unrealistic) case where the planetesimal can be considered to have all its mass concentrated at the center, one can use the Roche potential to show that in this case $\xi \simeq 2.1$.

All of this pertains to cases where the planetesimal under question is larger than a few $\mathrm{km}$ in size. Otherwise, for small bodies, they may simply be held together by solid-state forces amounting to tensile strengths that are large compared to the pressures imposed by gravity near the center of the body.

One can rewrite Eqn. (3) in a more convenient way for our problem by cubing both sides and solving for the critical density (i.e., below which the body is subject to tidal breakup), to find:

$$
\rho_{\text {crit }}=\frac{3 \xi^{3} M_{*}}{4 \pi s_{\text {crit }}^{3}}=\frac{3 \pi \xi^{3}}{G} \frac{1}{P_{\text {orb }}^{2}} \simeq 87(\xi / 2)^{3} \frac{1}{P_{\mathrm{hr}}^{2}} \mathrm{~g} \mathrm{~cm}^{-3}
$$

where in the second term on the right we made use of Kepler's 3rd law, and where in the final expression, $\xi$ has been normalized to a typical value of 2. For a period of 4.5 hours, as in the case of the innermost objects orbiting around WD 1145+017 we find critical densities of 7.9, 5.0, 2.6, and $1 \mathrm{~g} \mathrm{~cm}^{-3}$, depending on whether one uses Roche's value of $\xi$, the value of $\xi$ from the Roche potential, or other more recent values for $\xi$, respectively.

\section{Debris Rings:}

Once large rocky bodies have been tidally disrupted due to their close approaches to the white dwarf host, the result will be an eccentric ring of large chunks of debris (Veras et al. 2014, 2015). Through a process known as a "collisional cascade" (see, e.g., Kenyon and Bromley 2002, Kenyon and Bromley 2004; Kenyon et al. 2016; Wyatt et al. 2011) the sizable rocky bodies in this orbiting ring of debris undergo a sequence of collisions which eventually break down the few large objects into a very wide array of object sizes. These will range from planetesimals, to asteroids, rocks, pebbles, and dust, with a rough power-law size distribution.

In Figure 9 we show an artist's conception of what such a debris disk might look like. Note the size distribution ranging from asteroids down to dust.

At the current epoch we are apparently witnessing the debris disk in WD $1145+017$ after it has undergone a collisional cascade - which may, in fact, still be ongoing. The main goal of any comprehensive model would be to relate the properties of such a debris disk to the observational dipping activity in WD $1145+017$, the high-velocity gas absorption lines, and the excess NIR emission. The asymmetric transit profile and wildly variable transit depths observed from WD 1145+017 are qualitatively similar to transits of disintegrating planets around main sequence stars ("Disintegrating Rocky Exoplanets"; this Handbook), and it is believed that similar physical processes may be responsible for both types of transits, but with some significant differences. Perhaps the primary difference between the dusty-tailed planets around main-sequence stars and the situation in WD 1145+017 is the existence of numerous orbiting bodies in the white-dwarf system as opposed to a single more-substantive body orbiting the main-sequence stars. As we have seen, there are likely a dozen or more independently orbiting bodies in WD $1145+017$ of a size that can emit sufficient dust to block a significant fraction of the white dwarf's light. 


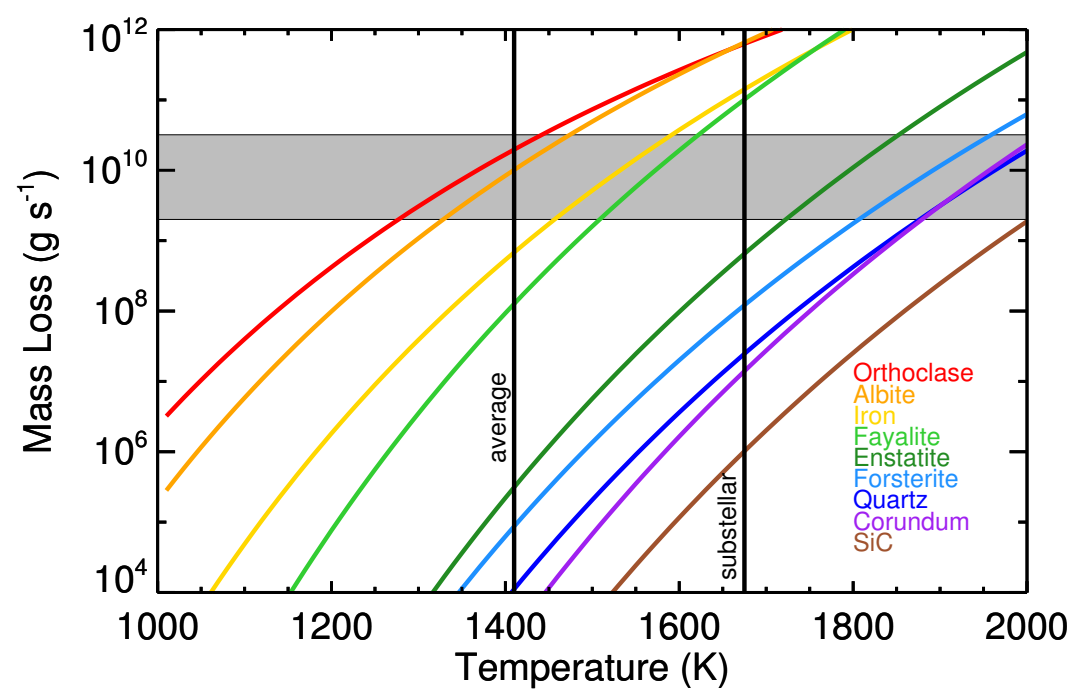

Fig. 10 Sublimation mass-loss rates for different minerals on the surface of a Ceres-sized body orbiting WD $1145+017$ (from Vanderburg et al. 2015).

\section{Explaining the Transits}

The transits around WD 1145+017 set it apart among all other polluted white dwarfs, and understanding what causes them is crucial to understanding the system as a whole. Among the various dips in flux, a number of them are uniquely identifiable, long lasting (i.e., for up to months), and of more or less constant shape and duration. Here, we describe three possible models of how the starlight is blocked.

\section{Continuous Dust Production Sublimating from Small Rocky Bodies}

The first model for explaining the transits of WD $1145+017$ is that dust is emitted continually from an orbiting rocky body at a relatively constant rate - merely giving the illusion of a permanent feature. This scenario requires a substantive underlying body in a fairly stable orbit to produce the deep and persistent transits observed. For tidally locked objects in nearly circular 4.5 hour orbits around WD 1145+017, the temperature of the object at the substellar point is about $1675 \mathrm{~K}$ and the average temperature over the dayside hemisphere is a bit lower at about $1410 \mathrm{~K}$. These temperatures are high enough that many minerals will sublimate. The vapor pressure, $p_{\text {vap }}$, and the knowledge that any vapors produced will freely stream away from the surface of such low-mass objects, allow us to calculate a mass loss flux $J$ from the surface of the body into a vacuum:

$$
J=\alpha p_{\text {vap }} \sqrt{\frac{\mathscr{M}}{2 \pi k_{B} T_{\text {eq }}}} \quad \text { where } \quad p_{\text {vap }}=\exp \left[-\frac{\mathscr{M} L_{\text {sub }}}{k_{B} T_{\text {eq }}}+b\right]
$$

and where $\mathscr{M}$ is the material's molecular mass, $L_{\text {sub }}$ is the material's latent heat of sublimation, $k_{B}$ is Boltzmann's constant, $T_{\mathrm{eq}}$ is the temperature of the body, $b$ is an empirically measured constant, and $\alpha$ is the "sticking coefficient" and is roughly 0.1 to 0.3 . Vanderburg et al. (2015) calculated mass loss rates for various different materials from a single Ceres sized object orbiting WD 1145+017 and found that several different materials and minerals, in particular orthoclase, albite, iron, and fayalite could sublimate and produce dust at the rates necessary to explain the transits of WD 1145+017. We show in Fig. 10 mass loss rates due to sublimation for a Ceres size object as a function of the surface equilibrium temperature for a variety of common minerals. Figure 8 of van Lieshout and Rappaport ("Disintegrating Rocky Exoplanets"; this Handbook) gives the sublimation rate as a function of planetesimal size for a fixed surface temperature of $2000 \mathrm{~K}$.

The realization from more recent large photometric monitoring campaigns (Gänsicke et al. 2016, Gary et al. 2016) that there are likely many smaller fragments producing deep transits and that these fragments are likely considerably 
smaller in order to coexist in very close orbits (Rappaport et al.2016) makes it more difficult to account for the deep transits via sublimation of refractory minerals. Vanderburg et al. (2015) calculated that the transits seen around WD $1145+017$ require mass-loss rates of order $10^{9} \mathrm{~g} \mathrm{~s}^{-1}$, and found that the mass loss fluxes calculated from Equation 5 could reproduce those mass loss rates if the surface area was that of a Ceres sized object with a radius of roughly 500 $\mathrm{km}$. However, if the fragments producing most of the transits at the A-period are actually closer in size to Halley's comet ( $\sim 5-10 \mathrm{~km}$ radius), as suggested by Rappaport et al. (2016), then their surface areas are roughly $10^{3}-10^{4}$ times smaller than Vanderburg et al. (2015) assumed. At the same mass loss fluxes, it therefore becomes difficult (but not out of the question) for sublimation of refractory elements to eject enough material to produce the observed transits. Some scenarios which might mitigate this problem and yield increased mass loss from small fragments include the presence of volatile elements (like water ice), which sublimates much faster than refractory materials like iron, or a higher surface area to mass ratio for the fragments than previously assumed. Alternatively, mass loss may not be completely driven by sublimation, and other processes such as volcanic activity or even collisions may contribute to the ejection of material causing the deep transits we see.

\section{Persistent dust from impulsive collisions}

The second scenario involves an impulsive ejection of dust. In this scenario, dust is ejected in discrete events. The wavelength dependence of the Mie absorption cross sections (and the corresponding inferred emissivities) for smaller dust particles causes these particles to be quickly heated to quite high temperatures (i.e., $\gtrsim 2000 \mathrm{~K}$ ) which leads to rapid sublimation. The result may be that the surviving grains are only those with sizes $\gtrsim$ few $\mu \mathrm{m}$. The calculated values of $\beta$, the ratio of radiation pressure forces to gravity, for these larger residual grains may be as low as $\beta \simeq 0.001$. Such small values of $\beta$ result in minimal radiation pressure to push the grains into a different orbit than the emitter, and, likewise, the orbital decay timescale due to the Poynting-Roberston effect may be much longer than the observation timescale. Therefore, in this scenario, the dust is released and simply hangs around the emitting asteroid for considerable intervals of time. The main effect in spreading such a dust cloud may be shearing due to its finite radial extent. If the cloud is 'ribbon-like', i.e., narrow in the radial direction, then it can avoid shearing. However, if it is extended by as much in the radial direction as it is perpendicular to the mid-plane (i.e., comparable to the size of the white dwarf), then the cloud would shear completely around the orbit within a couple of days.

\section{Azimuthal Asymmetry in a Dust Ring}

A final scenario invokes a quasi-permanent dust ring orbiting WD 1145+017 viewed edge on, which has azimuthal density asymmetries that cause differential extinction. When an over-dense region passes in front of the host star, it would cause a transit-like event. These asymmetries could not be self-gravitating - no reasonable masses (Gurri et al. 2017: Veras et al.2016a) could create a Hill sphere large enough to produce the dips we see - so the asymmetries would likely have to be driven by some orbiting shepherding objects, such as for some of the structures found in Saturn's rings (see e.g., Murray et al.|2005, 2008, Hyodo and Ohtsuki|2015). There are several observational arguments against this scenario. The first is that we often see transits, or diminishings of brightness, but not brightenings of the star. One would expect that the presence of over-densities that cause dimming events would also be accompanied by the presence of under-densities, which would cause brightening events when they pass in front of the host star. Second, the density waves in such a dust ring caused by shepherding bodies would be expected to show dip structures that have more manifest symmetries, and would not likely produce the isolated sharp narrow dip features that are sometimes observed in the lightcurves. Finally, it is not obvious how such an obscuring dust ring would change in overall density by more than an order of magnitude over the course of months as is seen (e.g., Fig. 7).

\section{Future Work}

The WD 1145+017 system is highly complex, rapidly evolving, and poses difficult observational and theoretical challenges. However, there are several paths forward to learning more about this object and the general process of how planetary material is tidally disrupted. In this section, we discuss both observational and theoretical studies which should be undertaken to further our understanding.

Continued Photometric Monitoring: The simplest future observations of WD 1145+017 are perhaps among the most important. In particular, it is crucial to continue observing WD 1145+017 photometrically with small to moderateclass telescopes. Continued photometric monitoring will allow us to track the transit activity level and determine the 
timescales on which the transit activity level changes. These observations are setting the first observational constraints on how long the tidal disruption of rocky bodies around white dwarfs last and how they progress, which are leading to new insights about the nature of the disrupted object (Veras et al.|2017). A more ambitious and far-reaching goal for photometric monitoring would be to detect and characterize objects orbiting in the sub-dominant B-F periods in the same way that objects in the A-period have been characterized to date. Now that photometric monitoring has revealed an object transiting at the sub-dominant B-period from $\mathrm{K} 2$, it is reasonable to believe that continued monitoring may reveal the frequency of transits of the B-F periods found in $\mathrm{K} 2$ relative to the numerous transits associated with the A-period, and lead to a stronger understanding of why the A-period experiences higher activity than the other periods. Finally, it would be good to carry out at least some photometry with large-aperture telescopes at shorter time-cadence to look for low-level and rapid timescale variability.

Continued High-Resolution Spectroscopic Monitoring: High resolution spectroscopy of WD 1145+017 has revealed intriguing patterns and changes in the circumstellar absorption features (Xu et al.2016, Redfield et al. 2016), but these observations are in general photon-starved. At 17th magnitude, high spectral resolution observations of WD 1145+017 are just barely possible on the short $(\lesssim 5$ minute) timescales of the photometric variability with 10 -meter class optical telescopes today. Future observations of WD 1145+017 with the next generation of efficient high-resolution spectrographs on 30-meter class telescopes should yield significantly superior signal-to-noise ratios on the timescales of transits, making a detailed examination of the short-timescale variability possible and revealing the interplay between the transiting dust and circumstellar gas.

UV spectroscopy: Ultraviolet spectroscopy of polluted white dwarfs often reveals the presence of additional elements in their spectra, and it is likely that WD $1145+017$ will be no exception. UV spectroscopy of WD 1145+017 is crucial to obtain while the Hubble Space Telescope (HST) is still operating in order to learn more about the composition of the disintegrating material and the circumstellar gas (which has previously been found in other white dwarfs in the ultraviolet, Gänsicke et al. 2012). These observations are forthcoming; an ongoing HST program led by Siyi Xu is obtaining ultraviolet spectroscopy of WD 1145+017 with the Cosmic Origins Spectograph (COS).

Gaia Parallax: ESA's Gaia mission will measure parallaxes of over a billion stars, including WD 1145+017. By comparing the precise trigonometric distance to WD 1145+017 with a photometric distance estimate, it will be possible to determine whether WD $1145+017$ is significantly extincted, and therefore to infer the presence of dust in the line of sight (even while out of transit).

Mid-Infrared Observations: Multi-wavelength observations of debris disks around white dwarfs can yield information about the extent and orientation of the disks (Jura 2003). This information is especially interesting in the case of WD 1145+017 because of the additional information we know about the system, in particular the orbital radii and inclination of the transiting debris. Naively, one would expect that for an edge-on system with an optically thick debris disk, where the orbits and debris disk are viewed at an inclination angle close to $90^{\circ}$, the luminosity of the disk would be relatively small, yet for the edge-on orbits at WD 1145+017 the disk luminosity is somewhat large.

Multi-wavelength photometric observations in the mid-infrared can yield information about the orientation and size of the debris disk that are not possible with current data (which only extends out to about 5 microns). Since the end of the Spitzer cryogenic mission in 2009, there are no telescope resources capable of detecting such faint sources at wavelengths beyond 5 microns, but the James Webb Space Telescope, scheduled to launch in 2018, will once again provide that capability at unprecedented sensitivity. Measuring the WD 1145+017 debris disk's spectral energy distribution into the mid-infrared could indicate a misalignment between the debris disk and the transiting material's orbits, or it could show that the disk is likely not optically thin (like the disk surrounding SDSS J155720.77+091624.6 Farihi et al.2016).

Finally, with regard to mid-IR observations, it is worth noting that measurements of the silicate feature at $10 \mu \mathrm{m}$ can directly reveal the presence of circumstellar silicates in dust grains (see, e.g., Jura et al. 2009).

Polarimetry: It would be quite challenging to measure possible polarization of light from WD 1145+017. Because there is not likely to be any preferred grain alignment via magnetic fields, any polarization would be due to scattering. If polarization can be measured, this would be quite useful in confirming the presence of dust and learning more about its spatial distribution.

Questions for Future Theoretical and Observational Investigations: Finally, here we list five questions which we believe are necessary to answer in the near future if we are to fully understand the WD 1145+017 system. While continued and further observations will help, these questions will likely require detailed calculations and study. 
- Is the dust production that feeds any of the dust clouds continuous, i.e., due to sublimation, or episodic, e.g., due to collisions of rocky bodies?

- If the dust production is episodic, then what keeps dust-cloud dip features orbiting coherently, in some cases for months? The dust clouds must be considerably larger than the Hill's sphere of any plausible body that is emitting the dust, and therefore the dust must be unbound.

- What causes the dramatic changes in dust cloud numbers and optical depth on timescales of days to years, like the change between observations by Vanderburg et al. (2015) and Croll et al. (2015), and those by Gänsicke et al. (2016) and Gary et al. (2016) a year later?

- What sets the apparent maximum flux decrease of $\sim 60 \%$ in the light of the white dwarf? Is this a result of changes in the optical depth of dust that covers essentially all of the white dwarf surface, or due to optically thick clouds that can, for some reason, never cover more than $60 \%$ of the white dwarf surface?

- How do the dust clouds become large enough to cause the large transit depths we see? In order to obscure large portions of the white dwarf the debris must be orbiting in different planes that are tilted by a range of angles spanning at least $\pm 1 / 2^{\circ}$. In turn, this implies velocity components that are perpendicular to the mean orbital plane of $\sim 3 \mathrm{~km} \mathrm{~s}^{-1}$, which are much too large for thermal speeds. This seems to require collisions. However, such high speeds would lead to rapid angular spreading of any resultant dust clouds that is much larger than observed. Finite sublimation lifetimes may help in regard to this latter issue.

Future observations and inquiries along these lines should improve our understanding of the many processes at work around polluted white dwarfs like WD $1145+017$ and contribute to our knowledge of the ultimate fate of planetary systems (including our own solar system) after their host stars retire from the main sequence.

Acknowledgements The authors are grateful to Bruce Gary, Siyi Xu, and Zach Berta-Thompson for quite helpful discussions and for sharing some of their unpublished results regarding WD 1145+017. We also acknowledge Ben Zuckerman for his insightful comments about the manuscript.

\section{References}

Alonso R, Rappaport S, Deeg HJ Palle E (2016) Gray transits of WD 1145+017 over the visible band. A\&A589:L6

Barber SD, Patterson AJ, Kilic M et al. (2012) The Frequency of Debris Disks at White Dwarfs. ApJ760:26

Becklin EE, Farihi J, Jura M et al. (2005) A Dusty Disk around GD 362, a White Dwarf with a Uniquely High Photospheric Metal Abundance. ApJ632:L119-L122

Berg C, Wegner G, Foltz CB, Chaffee FH Jr Hewett PC (1992) Spectroscopy and spectral types for 387 stellar objects from the large, bright QSO survey. ApJS78:409-421

Bonsor A, Mustill AJ Wyatt MC (2011) Dynamical effects of stellar mass-loss on a Kuiper-like belt. MNRAS414:930-939

Croll B, Dalba PA, Vanderburg A et al. (2015) Multiwavelength Transit Observations of the Candidate Disintegrating Planetesimals Orbiting WD 1145+017. ArXiv e-prints

Davidsson BJR (1999) Tidal Splitting and Rotational Breakup of Solid Spheres. Icarus142:525-535

Debes JH Sigurdsson S (2002) Are There Unstable Planetary Systems around White Dwarfs? ApJ572:556-565

Debes JH, Kilic M, Faedi F et al. (2012a) Detection of Weak Circumstellar Gas around the DAZ White Dwarf WD 1124-293: Evidence for the Accretion of Multiple Asteroids. ApJ754:59

Debes JH, Walsh KJ Stark C (2012b) The Link between Planetary Systems, Dusty White Dwarfs, and Metal-polluted White Dwarfs. ApJ747:148

Del Santo M, Nucita AA, Lodato G et al. (2014) The puzzling source IGR J17361-4441 in NGC 6388: a possible planetary tidal disruption event. MNRAS444:93-101

Farihi J, Gänsicke BT Koester D (2013) Evidence for Water in the Rocky Debris of a Disrupted Extrasolar Minor Planet. Science 342:218220

Farihi J, Parsons SG Gänsicke BT (2016) A Circumbinary Debris Disk in a Polluted White Dwarf System. ArXiv e-prints

Fontaine G Michaud G (1979) Diffusion time scales in white dwarfs. ApJ231:826-840

Friedrich S, Koester D, Christlieb N, Reimers D Wisotzki L (2000) Cool helium-rich white dwarfs from the Hamburg/ESO survey. A\&A363:1040-1050

Gänsicke BT, Marsh TR, Southworth J Rebassa-Mansergas A (2006) A Gaseous Metal Disk Around a White Dwarf. Science 314:1908

Gänsicke BT, Koester D, Farihi J et al. (2012) The chemical diversity of exo-terrestrial planetary debris around white dwarfs. MNRAS424:333-347

Gänsicke BT, Aungwerojwit A, Marsh TR et al. (2016) High-speed Photometry of the Disintegrating Planetesimals at WD1145+017: Evidence for Rapid Dynamical Evolution. ApJ818:L7

Gary BL, Rappaport S, Kaye TG, Alonso R Hambsch FJ (2016) WD 1145+017 Photometric Observations During 8 Months of High Activity. ArXiv e-prints 
Gurri P, Veras D Gänsicke BT (2017) Mass and eccentricity constraints on the planetary debris orbiting the white dwarf WD $1145+017$. MNRAS464:321-328

Holsapple KA Michel P (2006) Tidal disruptions: A continuum theory for solid bodies. Icarus 183:331-348

Hyodo R Ohtsuki K (2015) Saturn's F ring and shepherd satellites a natural outcome of satellite system formation. Nature Geoscience 8:686-689

Jura M (2003) A Tidally Disrupted Asteroid around the White Dwarf G29-38. ApJ584:L91-L94

Jura M, Farihi J Zuckerman B (2009) Six White Dwarfs with Circumstellar Silicates. AJ137:3191-3197

Kenyon SJ Bromley BC (2002) Collisional Cascades in Planetesimal Disks. I. Stellar Flybys. AJ123:1757-1775

Kenyon SJ Bromley BC (2004) Collisional Cascades in Planetesimal Disks. II. Embedded Planets. AJ127:513-530

Kenyon SJ, Najita JR Bromley BC (2016) Rocky Planet Formation: Quick and Neat. ApJ831:8

Koester D, Gänsicke BT Farihi J (2014) The frequency of planetary debris around young white dwarfs. A\&A566:A34

Manser CJ, Gänsicke BT, Koester D, Marsh TR Southworth J (2016) Another one grinds the dust: variability of the planetary debris disc at the white dwarf SDSS J104341.53+085558.2. MNRAS462:1461-1469

Murray CD, Chavez C, Beurle K et al. (2005) How Prometheus creates structure in Saturn's F ring. Nature437:1326-1329

Murray CD, Beurle K, Cooper NJ et al. (2008) The determination of the structure of Saturn's F ring by nearby moonlets. Nature453:739_ 744

Payne MJ, Veras D, Holman MJ Gänsicke BT (2016) Liberating exomoons in white dwarf planetary systems. MNRAS457:217-231

Perez-Becker D Chiang E (2013) Catastrophic evaporation of rocky planets. MNRAS433:2294-2309

Rappaport S, Levine A, Chiang E et al. (2012) Possible Disintegrating Short-period Super-Mercury Orbiting KIC 12557548. ApJ752:1

Rappaport S, Barclay T, DeVore J et al. (2014) KOI-2700b: A Planet Candidate with Dusty Effluents on a 22 hr Orbit. ApJ784:40

Rappaport S, Gary BL, Kaye T et al. (2016) Drifting asteroid fragments around WD 1145+017. MNRAS458:3904-3917

Redfield S, Farihi J, Cauley PW et al. (2016) Spectroscopic Evolution of Disintegrating Planetesimals: Minutes to Months Variability in the Circumstellar Gas Associated with WD 1145+017. ArXiv e-prints

Sanchis-Ojeda R, Rappaport S, Pallé E et al. (2015) The K2-ESPRINT Project I: Discovery of the Disintegrating Rocky Planet with a Cometary Head and Tail EPIC 201637175b. Astrophys J, submitted

Vanderburg A, Johnson JA, Rappaport S et al. (2015) A disintegrating minor planet transiting a white dwarf. Nature526:546-549

Veras D, Mustill AJ, Bonsor A Wyatt MC (2013) Simulations of two-planet systems through all phases of stellar evolution: implications for the instability boundary and white dwarf pollution. MNRAS431:1686-1708

Veras D, Leinhardt ZM, Bonsor A Gänsicke BT (2014) Formation of planetary debris discs around white dwarfs - I. Tidal disruption of an extremely eccentric asteroid. MNRAS445:2244-2255

Veras D, Leinhardt ZM, Eggl S Gänsicke BT (2015) Formation of planetary debris discs around white dwarfs - II. Shrinking extremely eccentric collisionless rings. MNRAS451:3453-3459

Veras D, Marsh TR Gänsicke BT (2016a) Dynamical mass and multiplicity constraints on co-orbital bodies around stars. MNRAS461:1413-1420

Veras D, Mustill AJ, Gänsicke BT et al. (2016b) Full-lifetime simulations of multiple unequal-mass planets across all phases of stellar evolution. MNRAS458:3942-3967

Veras D, Carter PJ, Leinhardt ZM Gänsicke BT (2017) Explaining the variability of WD $1145+017$ with simulations of asteroid tidal disruption. MNRAS465:1008-1022

Wyatt MC, Clarke CJ Booth M (2011) Debris disk size distributions: steady state collisional evolution with Poynting-Robertson drag and other loss processes. Celestial Mechanics and Dynamical Astronomy 111:1-28

Xu S Jura M (2014) The Drop during Less than 300 Days of a Dusty White Dwarf's Infrared Luminosity. ApJ792:L39

Xu S, Jura M, Dufour P Zuckerman B (2016) Evidence for Gas from a Disintegrating Extrasolar Asteroid. ApJ816:L22

Zhou G, Kedziora-Chudczer L, Bailey J et al. (2016) Simultaneous infrared and optical observations of the transiting debris cloud around WD 1145+017. MNRAS463:4422-4432

Zuckerman B Becklin EE (1987) Excess infrared radiation from a white dwarf - an orbiting brown dwarf? Nature330:138-140

Zuckerman B, Koester D, Melis C, Hansen BM Jura M (2007) The Chemical Composition of an Extrasolar Minor Planet. ApJ671:872-877

Zuckerman B, Melis C, Klein B, Koester D Jura M (2010) Ancient Planetary Systems are Orbiting a Large Fraction of White Dwarf Stars. ApJ722:725-736 